\title{
An Energy Detection Receiver Robust to Multi-User Interference for IEEE 802.15.4a Networks
}

\author{
Manuel Flury, Ruben Merz, Jean-Yves Le Boudec \\ EPFL, School of Computer and Communication Sciences \\ \{manuel.flury, ruben.merz, jean-yves.leboudec\}@epfl.ch
}

\begin{abstract}
Energy-detection receivers are appealing to IEEE 802.15.4a low data-rate networks because of their low complexity. With a reasonable energy consumption, these receivers can exploit the ranging capabilities and multipath resistance of impulse-radio UWB (IR-UWB). However, the performance of energy-detection receivers can be severely degraded by multi-user interference (MUI). One solution may be to coordinate access to the physical layer with an exclusion protocol. Unfortunately, this cannot prevent MUI due to uncontrolled activities in neighboring networks (e.g., several IEEE 802.15.4a piconets running in parallel). Hence, interference must be taken into account already in the design of the physical layer. In this paper, we present an IR-UWB receiver robust to MUI for IEEE 802.15.4a networks. Its architecture is based on energy detection. We also take into full account the different signaling structure between the preamble and the payload of IEEE 802.15.4a packets. In certain scenarios with MUI we found the packet error rate to be up to two orders of magnitude lower when compared to a traditional energy detection receiver that neglects MUI. Further, this significant performance improvement entails only a moderate increase in complexity.
\end{abstract}

\section{INTRODUCTION}

The IEEE 802.15.4 standard targets low data-rate wireless networks with extensive battery life and very low complexity. There are several options for the physical layer. The IEEE 802.15.4a amendment [1] specifies an impulse-radio ultrawide band (IR-UWB) physical layer that can operate over several bands of $500 \mathrm{MHz}$ (or $1.5 \mathrm{GHz}$ ) from about $3-10 \mathrm{GHz}$.

The IEEE 802.15.4a amendment allows for the implementation of either, a coherent receiver (e.g., a Rake receiver), or a non-coherent receiver (e.g., based on energy-detection). For IEEE 802.15.4a low data-rate networks, where energy consumption is of primary importance and devices should be inexpensive, energy-detection receivers are appealing. With a relatively low hardware complexity, these receivers can exploit the ranging capabilities and multipath resistance of IR-UWB. Compared to a Rake receiver, they operate at a lower sampling frequency, are robust to timing impairments, and do not need to perform a full channel estimation. Indeed, only the estimation of a channel power delay profile may be necessary [2], [3].

Unfortunately, the performance of energy-detection receivers is greatly affected by multi-user interference (MUI). In [4], the performance of an IEEE 802.15.4a compliant energy-detection receiver is analyzed in the presence of several transmitters using an Aloha medium access control (MAC)

The work presented in this paper was supported (in part) by the National Competence Center in Research on Mobile Information and Communication Systems (NCCR-MICS), a center supported by the Swiss National Science Foundation under grant number 5005-67322 layer (which is the mandatory MAC in IEEE 802.15.4a). It is shown that the packet error rate (PER) is severely increased by MUI, even at low data-rates.

One solution may be to prevent MUI by using a more sophisticated MAC to coordinate access to the physical layer. But, this cannot prevent MUI due to uncontrolled activities in neighboring networks (e.g., several IEEE 802.15.4a piconets running in parallel). Interference must thus be taken into account already in the design of the physical layer.

MUI in low data-rate IR-UWB networks is nonGaussian [5], and its probability density function exhibits an impulsive shape [6]. With non-Gaussian interference, [7], [8] suggest applying a non-linear function on the received signal prior to demodulation, for instance, a simple thresholding operation. Further, [9] shows the benefits of a thresholding structure on the achievable rate in IR-UWB networks.

Our contribution is a non-coherent receiver architecture robust to MUI and compliant with IEEE 802.15.4a. It is built around an energy detection receiver, and uses an adaptive nonlinearity, based on thresholding, to mitigate MUI. In order to correctly set the threshold, the power delay profile of the received signal needs to be properly estimated. This is done during the preamble that precedes the payload of an IEEE 802.15.4a packet. However, the structure of the signal in IEEE 802.15.4a differs between the preamble and the payload [1]. In the preamble, single pulses are sent, whereas in the payload, bursts of scrambled pulses are transmitted. Consequently, the power delay profile of the payload signal is time-varying due to the scrambling operation; it is impossible to estimate it with a classical energy-detection receiver. Our receiver overcomes this issue. It does so in a way that is robust to MUI and only with a moderate increase in complexity. Note that we concentrate on a robust power delay profile estimation and data decoding. We do not consider robust timing acquisition in this paper. Due to the lack of space, it is left out for further studies. But we point out the work in [10], [11] that already suggest potential solutions for robust timing acquisition.

The system model and assumptions are given in Section II. We describe the architecture of our receiver in Section III, and we evaluate its performance in Section IV. We conclude the paper in Section $\mathrm{V}$.

\section{System Model And Assumptions}

We consider an IEEE 802.15.4a IR-UWB physical layer and focus on non-coherent reception with binary pulse position modulation (BPPM) and time-hopping. 
An IEEE 802.15.4a packet consists of two parts: a preamble followed by a payload. The preamble is known to the receiver and used for packet detection and timing acquisition. One peculiarity of IEEE 802.15.4a is the different signaling format used in the preamble and the payload. The preamble consists of a sequence of single, amplitude modulated pulses. In contrast, each symbol of the payload is composed of a short, continuous burst of $L_{b}$ pulses with pseudo-random polarity.

The main unit of time of a packet is a chip of duration $T_{c}$. During the preamble, pulses can only be sent at regular intervals, every $L$-th chip. The received signal after filtering with a bandpass filter of bandwidth $B$ is then given by

$$
r^{\text {pre }}(t)=\sum_{i} s_{i} \cdot h\left(t-i L T_{c}\right)+n(t)
$$

where $h(t)$ is the unknown channel response (including the transmitted waveform, the response of the multipath channel and the bandpass filter), $n(t)$ accounts for thermal noise and MUI, and $s_{i} \in\{-1,0,+1\}$ is a ternary preamble code. It is assumed that the duration of $h(t)$ is shorter than $L T_{c}$ to prevent inter-symbol interference (ISI).

The received signal during the payload is given by

$r(t)=\sum_{i} \sum_{j=0}^{L_{b}-1} b_{i j} \cdot h\left(t-i T_{f}-c_{i} L_{b} T_{c}-a_{i} T_{f} / 2-j T_{c}\right)+n(t)$

where $T_{f}$ is the duration of a symbol, $a_{i} \in\{0,1\}$ is the $i$ th symbol of the payload (which is unknown), $c_{i}$ denotes the time-hopping sequence and $b_{i j} \in \pm 1$ is the pseudo-random polarity of the $j$-th pulse of the $i$-th symbol specified by the scrambling sequence. Both $c_{i}$ and $b_{i j}$ are known to the receiver. Without loss of generality, we consider only the first symbol (i.e., we set $i=0$ and drop the index $i$ ) and assume $c_{0}=0$, yielding

$$
r(t)=\sum_{j=0}^{L_{b}-1} b_{j} \cdot h\left(t-a T_{f} / 2-j T_{c}\right)+n(t) .
$$

\section{Architecture of the Robust Receiver}

We assume a classical energy-detection receiver performing a squaring operation followed by an integrator sampled at a rate $1 / T$ and yielding the discrete time signals

$y_{m, i}^{\text {pre }}=\int_{m T+i L T_{c}}^{(m+1) T+i L T_{c}}\left[r^{p r e}(t)\right]^{2} d t, \quad y_{m}=\int_{m T}^{(m+1) T} r(t)^{2} d t$

depending on whether we are receiving the preamble or the payload and where $m=0, \ldots, N-1$, with $N=L T_{c} / T$.

\section{A. Optimal Decision Rule for Burst Transmissions}

Assuming that $n(t)$ is purely AWGN with power spectral density $N_{0} / 2$ bandlimited to $B$, the samples $y_{m}$ are independently and identically distributed according to a non-central chi-square distribution with $2 B T$ degrees of freedom and noncentrality parameter $\lambda_{m, a}=\frac{1}{N_{0} / 2} p_{m, a}$ [12], i.e.

where

$$
y_{m} \sim N C \chi_{2 B T, \lambda_{m, a}}^{2}
$$

$$
p_{m, a}=\int_{m T}^{(m+1) T}\left[\sum_{j=0}^{L_{b}-1} b_{j} \cdot h\left(t-a T_{f} / 2-j T_{c}\right)\right]^{2} d t
$$

Let $N_{f}=T_{f} / T$ and observe that $p_{m, 1}=p_{m-N_{f} / 2,0}$. Thus, we can simplify the notation by introducing $p_{m} \doteq p_{m, 0}$. Under the above assumptions, the optimal decision rule according to the maximum likelihood criterion is found as

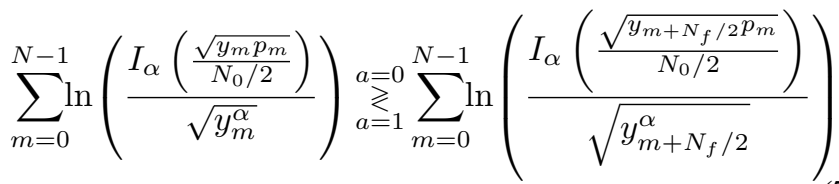

where $\alpha=B T-1$ and $I_{\alpha}(y)$ denotes the $\alpha$-th order modified Bessel function of the first kind. Equation (7) can be linearly approximated [3] resulting in a more practical decision rule

$$
\sum_{m=0}^{N-1} y_{m} \cdot p_{m} \underset{a_{0}=1}{\stackrel{a_{0}=0}{\gtrless}} \sum_{m=0}^{N-1} y_{m+N_{f} / 2} \cdot p_{m}
$$

Hence, the optimal detector applies a weighting function with coefficients $p_{m}$ prior to comparing the energies in the first and second half of a data frame. With $L_{b}>1,(6)-(8)$ give us a generalization of the result found in [3]. With $L_{b}=1$ the weighting function reduces to the one found in [3]. How to estimate the weights $p_{m}$ is shown in Section III-C.

\section{B. A Thresholding Mechanism to Reduce the Impact of MUI}

In the presence of MUI, the assumption of $n(t)$ being AWGN does not generally hold [5]. Therefore, we additionally use a thresholding mechanism to mitigate MUI. As stated before, $y_{m} \sim N C \chi_{2 B T, \lambda_{m}}^{2}$ if the interference is only AWGN. If MUI occurs, the distribution of $y_{m}$ will deviate from (5). Accordingly, the receiver can calculate a threshold

$$
\nu_{m}=\frac{N_{0}}{2} F_{N C \chi_{2 B T, \lambda_{m}}^{2}}^{-1}\left(1-P_{F A}\right)
$$

where $F_{N C \chi_{2 B T, \lambda m}^{2}}(x)$ is the cumulative distribution function of the non-central chi-square distribution and $P_{F A}$ is a small false-alarm probability that allows us to set the sensitivity of the threshold. The threshold $\nu_{m}$ can be made adaptive by adjusting $P_{F A}$.

Prior to the decision process, the receiver applies a nonlinear threshold operation governed by $\nu_{m}$ to the received samples in order to mitigate or even reject high interference terms. Different non-linear operations are possible. The one we found to work best is to set samples, above the threshold, to the value of the corresponding weight $p_{m}$

$$
y_{m}= \begin{cases}y_{m} & \forall m: y_{m} \leq \nu_{m} \\ p_{m} & \forall m: y_{m}>\nu_{m}\end{cases}
$$

As can be seen from (9), the threshold $\nu_{m}$ depends on the weights $p_{m}$ as well as on the thermal noise level. How to estimate these quantities is shown in Section III-C.

\section{Estimation of the Weighting Coefficients $p_{m}$}

To show how the weighting coefficients $p_{m}$ can be estimated from the preamble, we rewrite (6)

$$
\begin{aligned}
p_{m} & =\sum_{j=0}^{L_{b}-1} b_{j}^{2} \int_{m T}^{(m+1) T} h^{2}\left(t-j T_{c}\right) d t \\
& +2 \sum_{j=0}^{L_{b}-1} \sum_{k=j+1}^{L_{b}-1} b_{j} b_{k} \int_{m T}^{(m+1) T} h\left(t-j T_{c}\right) h\left(t-k T_{c}\right) d t .
\end{aligned}
$$


By applying the change of variables $s \doteq t-j T_{c}$ and introducing $K \doteq T_{c} / T$, we obtain

$$
\begin{aligned}
p_{m} & =\sum_{j=0}^{L_{b}-1} \int_{(m-j K) T}^{(m-j K+1) T} h^{2}(s) d s \\
& +2 \sum_{j=0}^{L_{b}-1} \sum_{k=j+1}^{L_{b}-1} b_{j} b_{k} \int_{(m-j K) T}^{(m-j K+1) T} h(s) h\left(s-(k-j) T_{c}\right) d s \\
& =\sum_{j=0}^{L_{b}-1} w_{m-j K}^{(0)}+2 \cdot \sum_{j=0}^{L_{b}-1} \sum_{k=i+1}^{L_{b}-1} b_{j} b_{k} \cdot w_{m-j K}^{(k-j)}
\end{aligned}
$$

where $w_{m}^{(l)}, l=1, \ldots, L_{b}-1$ is given by

$$
w_{m}^{(l)}=\int_{m T}^{(m+1) T} h(t) h\left(t-l \cdot T_{c}\right) d t
$$

To estimate $p_{m}$, we have to estimate the parameters $w_{m}^{(l)}$. Note that $w_{m}^{(0)}$ represents the energy-delay profile of the channel and corresponds to the weight applied in [3]. Plugging (1) into (4) and taking expectations yields

$$
\mathbb{E}\left[y_{m, i}^{p r e}\right]=s_{i}^{2} \underbrace{\int_{m T}^{(m+1) T} h^{2}(t) d t}_{w_{m}^{(0)}}+\underbrace{\mathbb{E}\left[\int_{0}^{T} n^{2}(t) d t\right]}_{\bar{n}}
$$

This suggests that we can get an estimate $\hat{\bar{n}}$ of $\bar{n}$ from the sample mean of the samples $y_{m, i}^{p r e}$ for which $s_{i}=0$. We can futher get an estimate $\hat{w}_{m}^{(0)}$ of $w_{m}^{(0)}$ by first averaging over the samples $y_{m, i}^{p r e}$ for which $s_{i} \neq 0$ and then subtracting $\hat{\bar{n}}$. Note that using the sample mean as a practical estimator for (15) is not robust if $n(t)$ is non-Gaussian. We address this issue in Section III-D.

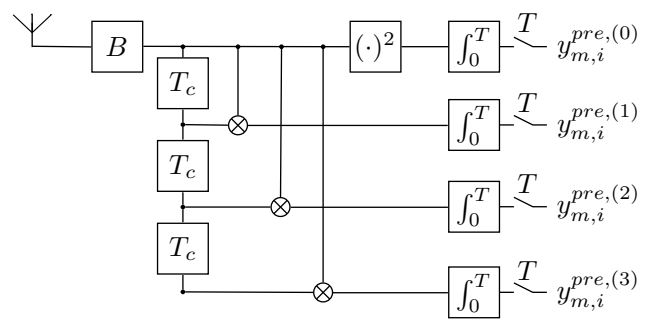

Fig. 1. Proposed receiver structure in the case of payload signaling with bursts of four pulses $\left(L_{b}=4\right)$. The additional branches are needed only during estimation of the parameters $w_{m}^{(l)}$ given in (14). They are not needed for data decoding where only the upper branch is required. Hence, their impact on power consumption is minimal.

Also note that there is no way for $w_{m}^{(l)}, l \neq 0$, to be estimated by an existing energy-detection receiver architecture. Consequently, equations (13) and (14) not only define a new weighting function but also show the necessity for a new receiver structure that allows for the estimation of the parameters $w_{m}^{(l)}$. To this end, we propose a receiver employing $L_{b}-1$ additional branches with respect to a classical energy-detection receiver as shown in Figure 1. However, the additional branches are only needed during the preamble, while estimating the parameters $w_{m}^{(l)}$. During the other phases of packet reception, synchronization and decoding, the additional circuitry is not used. The added complexity and power consumption should thus be moderate. This also limits the additional memory requirements of this more sophisticated receiver. The $l$-th additional branch delays the received signal by $l T_{c}$ and multiplies the received signal with this delayed version. The resulting signal is then integrated and sampled to yield the samples $y_{m, i}^{\text {pre, }(l)}$.

The quantity $\hat{w}_{m}^{(0)}$ can be obtained from the undelayed branch $y_{m, i}^{\text {pre, }}(0)$ (see Figure 1) as before according to (15). $\hat{w}_{m}^{(l)}, l \in\left\{1, \ldots, L_{b}-1\right\}$ can be obtained in a similar way from the $l$-th branch, thanks to the observation that

$$
\mathbb{E}\left[y_{m, i}^{p r e,(l)}\right]=s_{i}^{2} \underbrace{\int_{m T}^{(m+1) T} h(t) h\left(t-l \cdot T_{c}\right) d t}_{w_{m}^{(l)}}
$$

From the parameters $\hat{w}_{m}^{(l)}, l \in\left\{0, \ldots, L_{b}-1\right\}$ an estimate of the weights $\hat{p}_{m}$ can be directly calculated using (13) under the condition that $K=T_{c} / T$ is an integer greater than or equal to one or in other words $T \leq T_{c}$.

If $K<1, p_{m}$ can still be calculated but the integrators in Figure 1 have to be replaced by a bank of time-delayed parallel integrators to obtain the shifted parameters $w_{m-j K}^{(l)}$. Due to space restrictions, we cannot go into details of this alternative design. Nevertheless, a tradeoff between a lower possible sampling frequency and additional required circuitry is possible.

In addition to the weights $p_{m}$, we also have to estimate the power spectral density $N_{0} / 2$ of the noise; it is used to calculate the threshold $\nu_{m}$. An estimate can be obtained from $\hat{\bar{n}}$ given in (15) as $\frac{\hat{N}_{0}}{2}=\frac{\hat{\bar{n}}}{2 B T}$.

\section{Robust Parameter Estimation Using Order Statistics}

If done according to (15) and (16), the estimation of $\hat{w}_{m}^{(l)}$ and $\hat{\bar{n}}$ is not robust to MUI if the number of samples used to calculate the sample mean is small and/or if the interference level is very high.

A more robust option in these cases is to use order statistics to calculate these estimates by replacing the sample mean by the sample median which is more robust to outliers. If $\hat{\bar{n}}$ has been calculated in this way, the esimation of the power spectral density of the noise is replaced by $\frac{\hat{N}_{0}}{2}=\frac{\hat{\bar{n}}}{2 B T-2 / 3}$.

\section{Performance Evaluation}

In this section, we evaluate the performance of an IEEE 802.15.4a physical layer that uses our robust receiver (described in Section II) in the presence of MUI. The main performance metric is the packet error rate (PER).

We perform a packet-based simulation. There is one receiver and $N_{u}$ transmitters. We simulate the estimation of the power delay profile of the channel followed by data decoding (with the $(63,55)$ Reed-Solomon code). We assume that synchronization always succeeds. Each transmitter has a queue with packet arrival rate $\lambda_{i}, i=0,1, \ldots, N_{u}-1^{1}$. We send 1014 bits per packet. We have $T_{c}=T=2 \mathrm{~ns}(500 \mathrm{MHz}$ sampling

\footnotetext{
${ }^{1}$ For each packet reaching the front of the queue, we draw a backoff time according to the IEEE 802.15.4a procedure with the backoff exponent set to its maximum value. When the backoff expires, the packet is transmitted on the channel. A utilization of $100 \%$ corresponds to roughly $\lambda=200$ packets/s.
} 

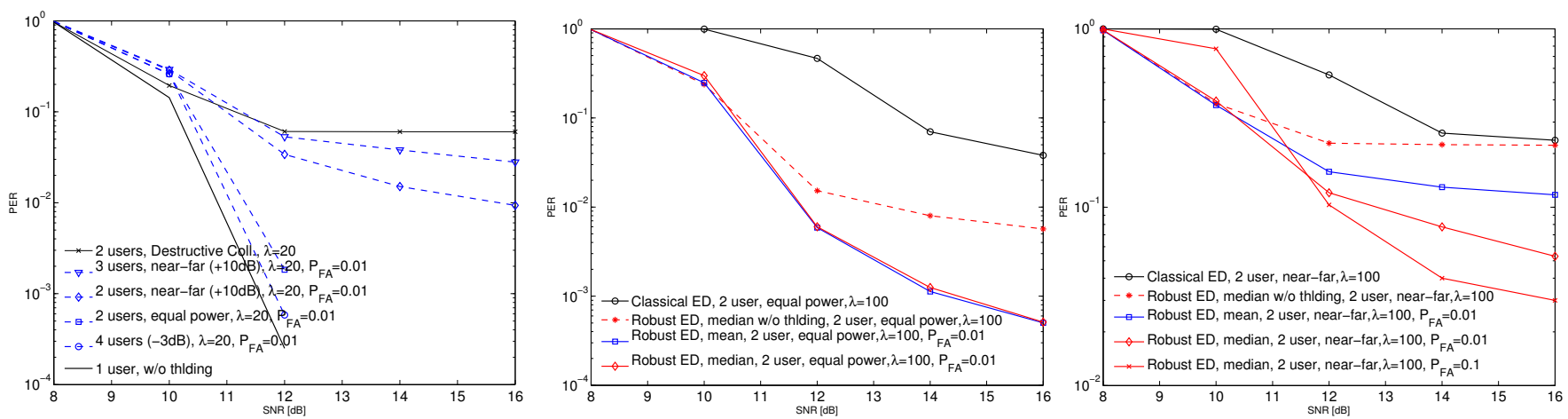

Fig. 2. PER with MUI in a low traffic case $(\lambda=$ Fig. 3. Comparison of a classical receiver (performing weighting but no burst adaptation and no 20 packet/s). With equal or lower power interferers, thresholding) to our receiver $(\lambda=100$ packets/s). On the left we show simulations with one equal performance is close to single user. Even in a harsh power interferer. The performance improvement is roughly two orders of magnitude in PER. On the near-far scenario the receiver shows some capture right we show a near-far scenario. In this case the sample mean is not robust to MUI. Also, by using effect when compared to the worst-case "Destructive a more aggressive threshold $\left(P_{F A}=0.1\right)$, we can push the performance improvement to one order of Collisions" model. magnitude in PER. Both figures underline that burst adaptation and thresholding are required.

frequency). The mandatory frequency band 3 is used, where the two possible preamble codes are code 5 and 6 [1]. The transmitter of interest uses code 5 and the others use code 6 . We use the IEEE 802.15.4a channel model 1 [13]. We simulate the physical layer with an accuracy of 100 ps (a simulation sampling frequency of $10 \mathrm{GHz}$ ).In our simulations, the signal to noise ratio (SNR) is $\mathrm{SNR}=\frac{E_{p}}{N_{0}}$ where $E_{p}$ is the received energy per pulse (after the convolution of the pulse with the impulse response of the channel).

In Figure 2, we show the PER obtained with our receiver, using the sample median for power delay profile estimation and the threshold set according to $P_{F A}=0.01 . \lambda=20$ packet/s for scenarios with $N_{u}$ from 1 to 4 and various received powers. We also show a "Destructive Collisions" model. With this model, a packet is lost whenever there is more than one active transmission at the same time and single user performance is assumed when there is only the transmitter of interest. For both cases with two equal power users and with three additional weaker-power users (at $-3 \mathrm{~dB}$ ), the PER is close to the one of the single user scenario. In near-far cases (the additional transmitters have $10 \mathrm{~dB}$ higher power), there is still a significant performance increase compared to the worst case "Destructive Collisions" curve. Note that with a conventional receiver where no thresholding is done, the near-far cases correspond to the "Destructive Collisions" curve already at a lower rate of $\lambda=10 \mathrm{pkts} / \mathrm{s}$ [4].

In Figure 3, we consider an equal power (left) and a near-far (right) scenario: first, the performance of a classical energydetection receiver without thresholding [3], where the weighting function does not take the bursts into account; second, an energy-detection receiver with the weighting function taking the bursts into account but without thresholding; third, our receiver, using the sample mean or the sample median. For both scenarios, $\lambda=100$ packet/s. Clearly, the sample mean is not robust to interference in near-far cases. Also, both a thresholding mechanism and taking into account the bursts are necessary. Our receiver achieves a performance improvement of up to roughly two orders of magnitude in PER in the equal power case and of one order of magnitude in the near-far case. We observed that this result does not seem to depend on $\lambda$; we found it to be the same for $\lambda=20,100,200$ packets/s.

\section{CONClusion}

We have presented a robust energy-detection receiver, yielding excellent performance, even in near-far scenarios. The complexity increase with respect to a classical energydetection receiver is minimal; it is necessary only during the estimation of the power delay profile of the channel. For future work, we plan to include a robust packet detection and synchronization scheme. We also plan to have the parameter $P_{F A}$ adaptive to the level of interference.

\section{REFERENCES}

[1] "IEEE P802.15.4a/D7 (amendment of IEEE std 802.15.4), part 15.4: Wireless medium access control (MAC) and physical layer (PHY) specifications for low-rate wireless personal area networks," Jan. 2007.

[2] M. Weisenhorn and W. Hirt, "ML receiver for pulsed UWB signals and partial channel state information," in Ultra-Wideband, 2005. ICU 2005. 2005 IEEE International Conference on, 2005, pp. 6 pp.+.

[3] A. A. D'Amico, U. Mengali, and E. Arias-De-Reyna, "Energy-detection UWB receivers with multiple energy measurements," IEEE Trans. Wireless Commun., vol. 6, no. 7, pp. 2652-2659, 2007.

[4] M. Flury, R. Merz, J.-Y. le Boudec, and J. Zory, "Performance evaluation of an IEEE 802.15.4a physical layer with energy detection and multi-user interference," in Ultra-Wideband, 2007. ICUWB 2007. IEEE International Conference on, September 2007, pp. 663-668.

[5] G. Durisi and G. Romano, "On the validity of Gaussian approximation to characterize the multiuser capacity of UWB TH PPM," in Ultra Wideband Systems and Technologies, 2002. Digest of Papers. 2002 IEEE Conference on, 2002, pp. 157-161.

[6] A. R. Forouzan, M. Nasiri-Kenari, and J. A. Salehi, "Performance analysis of time-hopping spread-spectrum multiple-access systems: uncoded and coded schemes," IEEE Trans. Wireless Commun., no. 4, pp. 671681, 2002.

[7] A. Spaulding and D. Middleton, "Optimum reception in an impulsive interference environment-part I: Coherent detection," IEEE Trans. Commun., vol. 25, no. 9, pp. 910-923, 1977.

[8] _ - "Optimum reception in an impulsive interference environmentpart II: Incoherent reception," IEEE Trans. Commun., vol. 25, no. 9, pp. 924-934, 1977.

[9] R. Knopp and Y. Souilmi, "Achievable rates for uwb peer-to-peer networks," in Communications, 2004 International Zurich Seminar on, 2004, pp. 82-85.

[10] A. El Fawal and J.-Y. Le Boudec, "A robust signal detection method for ultra wide band (UWB) networks with uncontrolled interference," IEEE Trans. Microw. Theory Tech., vol. 54, no. 4, pp. 1769-1781, June 2006.

[11] Z. Sahinoglu and I. Guvenc, "Multiuser interference mitigation in noncoherent UWB ranging via nonlinear filtering," EURASIP Journal on Wireless Communications and Networking, pp. 1-10, 2006.

[12] H. Urkowitz, "Energy detection of unknown deterministic signals," Proc. IEEE, vol. 55, no. 4, pp. 523-531, 1967.

[13] "IEEE 802.15.4a channel model - final report, document 04/662r0," http://www.ieee802.org/15/pub/TG4a.html, November 2004. 